INRA Prod. Anim., 2008, 21 (5), 419-426

\section{La politique de l'eau : approche économique et application à la pollution des élevages}

P. LE GOFFE

INRA, Agrocampus Ouest, UMR1302 Structures et Marchés Agricoles, Ressources, Territoires,

F- 35011 Rennes, France

Courriel :Philippe.Legoffe@agrocampus-ouest.fr

En Bretagne, la politique de l'eau donne des résultats décevants au regard des sommes investies. Ceci s'explique largement par le refus d'appliquer le principe pollueur-payeur, malgré les injonctions de la Directive Cadre sur l'Eau et l'exemple des pays du nord de l'Europe.

Dans «L'environnement en France», l'Institut français de l'environnement dresse le bilan de la pollution de l'eau (IFEN 2006). Alors que les rejets ponctuels dans les cours d'eau ont nettement diminué depuis une vingtaine d'année, qu'il s'agisse des rejets de station d'épuration urbaine ou de rejets industriels, les pollutions diffuses restent les plus préoccupantes. Il semble cependant qu'on assiste pour la première fois à une stabilisation des nitrates agricoles dans les eaux superficielles de Bretagne, même si leur concentration continue à augmenter dans les eaux souterraines d'autres régions (bassin parisien notamment). En Bretagne, Aurousseau (2008) confirme à partir de données récentes qu'il semble que la qualité des eaux s'améliore depuis le début des années 2000, mais que cette amélioration reste modérée car on ne retrouve jamais une qualité comparable à celle observée avant 1990 .

Cette stabilisation ou amélioration de la qualité des eaux en Bretagne est le fruit de politiques publiques combinant réglementation et incitations économiques, c'est-à-dire les efforts des producteurs, mais aussi ceux de l'Etat et des collectivités territoriales. Ceci explique pourquoi l'évaluation de ces politiques publiques ne peut se faire à la seule aune de leur efficacité environnementale, mitigée ici, mais doit aussi intégrer leur coût pour la société et les contribuables en particulier, c'est-à-dire leur efficacité ou efficience économique. Deux questions se posent dans un premier temps : 1) l'amélioration peut-elle être obtenue à un coût inférieur ? et 2) même minimisé, le coût des mesures à l'origine de l'amélioration est-il disproportionné par rapport aux bénéfices escomptés ? Dans un deuxième temps, une autre question est de se demander qui, des producteurs ou des consommateurs-contribuables, va payer les mesures.

En France, la politique de l'eau est généralement considérée comme coûteuse pour le budget de l'Etat et des collectivités. S'agissant de la Bretagne et de la mise aux normes des élevages, les aides prévues par l'Etat et l'agence de l'eau au titre du programme de mâ̂trise des pollutions agricoles (PMPOA), débuté en 1993, avoisinent le milliard d'euros. Le coût du plan d'action pour un développement pérenne de l'agriculture et de l'agroalimentaire en Bretagne et pour la reconquête de la qualité de l'eau, arrêté en 2002 pour la période 2002-2006, a été estimé à 473 millions d'euros (Anonyme 2002). Le programme Bretagne Eau Pure 3 représente 100 millions d'euros sur 7 ans. Enfin, l'Etat a prévu 60 millions d'euros sur cinq ans, pour financer les mesures arrêtées dans le cadre des bassins versants en contentieux avec l'Union Européenne, qui couvrent 4\% de la Surface Agricole Utile (SAU) de la Bretagne1. A ces aides publiques, il faut ajouter les coûts directement supportés par les éleveurs, dans la mesure où les aides couvrent une proportion du montant des investis- sements comprise entre un tiers et deux tiers. L'ensemble de ces financements représente le coût pour la société.

La Cour des comptes (1997 et 2002) et le Commissariat général au Plan (1997) ont remis plusieurs avis sur la politique de l'eau dans les années précédant la réforme de la loi sur l'eau : rapports sur les agences de l'eau, rapport sur la politique de préservation de la ressource en eau destinée à la consommation humaine (VilleyDesmeserets et al 2001), rapport sur la préservation de la ressource en eau face aux pollutions d'origine agricole, le cas de la Bretagne. Certaines des critiques qu'ils émettaient alors sont toujours valables aujourd'hui, malgré la réforme de 2006 : mauvaise application du principe pollueur-payeur, redevances peu incitatives et peu liées aux rejets et prélèvements, cycle pollution-dépollution préféré à la réduction à la source, projets surdimensionnés, coûts non minimisés.

Ces dysfonctionnements de la gestion de l'eau dans notre pays sont d'autant plus surprenants qu'il existe depuis 2000 un cadre européen de la politique de l'eau, décrit dans la directive cadre sur l'eau (Anonyme 2000). Certes, la Directive Cadre sur l'Eau (DCE) place les questions environnementales au premier plan, en affirmant la dimension patrimoniale de l'eau et en fixant l'objectif de bon état des eaux à l'horizon 2015. Cependant, elle représente un progrès par rapport aux textes anté-

${ }^{1}$ L'extension de ces mesures à toute la Bretagne représenterait un budget de 1,5 milliard d'euros sur cinq ans. 
rieurs, car c'est la première directive environnementale qui comporte également des principes économiques issus du corpus théorique de l'économie de l'environnement (Courtecuisse et al 2002). En ce sens, la DCE est un véritable instrument du développement durable.

L'idée de cet article est que, contrairement à ce qu'on observe dans les pays du nord de l'Europe, notre politique de l'eau n'est pas conforme aux principes économiques de la DCE. C'est ce qui explique que la qualité de l'eau ne s'améliore pas suffisamment, d'où des contentieux européens persistants, d'autant moins que les sommes engagées pour réduire les pollutions sont importantes et qu'elles comportent une part élevée d'argent public. L'article comporte trois parties. Dans la première partie, on montre à la lumière de l'économie de l'environnement comment l'association des objectifs écologiques et des principes économiques réalisée par la DCE permet de conférer à la politique de l'eau une double efficacité environnementale et économique. Pour illustrer l'idée de l'article, nous avons choisi de présenter dans la deuxième partie la politique française de maîtrise des pollutions des élevages. Il s'agit d'une branche relativement complexe de la politique de l'eau, qui a connu de nombreuses vicissitudes, ce qui en fait un «cas d'école». La dernière partie est consacrée à montrer en quoi les dispositifs équivalents adoptés dans les pays du nord de l'Europe sont davantage conformes aux principes de la DCE. En conclusion, on s'inspire du cadre européen et des exemples étrangers pour proposer des pistes d'amélioration de notre dispositif de maîtrise des pollutions des élevages.

\section{1 / La Directive Cadre sur l'Eau et l'économie}

\section{1 / L'optimum de Pareto}

La DCE fait référence à deux grands principes économiques qui sont au cœur des théories de l'économie publique et de l'économie de l'environnement. Ces théories développent une approche anthropocentrée de l'environnement, en ce sens que l'environnement est apprécié à travers le bien-être qu'il procure à l'homme. L'amélioration ou la dégradation de l'environnement donnent lieu à des gains ou des pertes de bien-être, appelées respectivement bénéfices ou dommages environnementaux, que les économistes tentent d'évaluer monétairement en ayant recours à différentes méthodes spécifiques.

Le premier principe est celui qui considère l'optimum de Pareto comme un état idéal de l'économie. L'optimum de Pareto est défini comme cet état où on ne peut augmenter le bien-être d'un agent sans diminuer celui d'un autre. Si on considère que le bien-être des différents agents économiques est comparable et additif et que les gagnants peuvent compenser les perdants, rechercher l'optimum revient à maximiser la valeur collective ou sociale nette, c'est-à-dire les bénéfices tirés des activités productives diminués des dommages à l'environnement. Dans le cas où une activité de dépollution est introduite, on cherchera à minimiser la somme des coûts de dépollution et des dommages à l'environnement, c'est-àdire les coûts sociaux. Cette approche conduit au «bon» niveau d'environnement, qui doit être visé par le planificateur social. Pour savoir empiriquement si une mesure augmente le bien-être collectif et est donc socialement désirable, on procède alors à une analyse coûts-avantages pour mesurer et comparer les coûts et les avantages de la mesure.

La figure 1 applique ces concepts au raisonnement de la densité animale, mesurée en abscisse, les valeurs économiques par unité de surface étant portées en ordonnée. L'optimum privé des producteurs n'est pas visible sur le graphique, car on considère qu'il faut une densité animale très élevée pour observer un plafonnement, voire une baisse du bénéfice privé des producteurs par unité de surface, par exemple sous l'effet de facteurs sanitaires. Les courbes $\mathrm{D}_{1}$ et $\mathrm{D}_{2}$ correspondent aux dommages environnementaux observés respectivement en zone banale et en zone sensible. En zone banale, le dommage essentiellement lié à la pollution de l'eau apparaît lorsque les apports nutritifs d'origine animale excèdent la capacité d'exportation des cultures. En zone sensible, telles les zones périurbaines, littorales ou écologiques patrimoniales, le dommage recouvre les pollutions mais aussi les nuisances paysagères et olfactives, ce qui explique qu'il soit ressenti pour des densités animales plus faibles. La valeur collective maximale est observée pour une densité Q1 en zone banale, qui correspond sur le graphique à la liaison de la production animale au sol, et pour une densité inférieure Q2 en zone sensible. Ceci plaide pour une différenciation des normes selon les zones, qui porterait peut-être sur la densité animale, mais aussi sur le type d'élevage (industriel ou pas).

L'analyse coûts-avantages apparaît explicitement dans la DCE, qui précise que «la communauté doit tenir compte, lors de l'élaboration de sa politique de l'environnement... des avantages et des coûts qui peuvent résulter de l'action ou de l'absence d'action». La notion de coût démesuré ou disproportionné est évoquée à plusieurs reprises pour justifier la dérogation à l'objectif de bon état écologique des eaux. C'est notamment le cas pour la désignation des masses d'eau de surface en catégorie artificielle ou fortement modifiée ${ }^{2}$. Dans ce cas, les impossibilités techniques ou les coûts disproportionnés empêchent d'obtenir une amélioration environnementale à un coût raisonna-

Figure 1. Le raisonnement économique de la densité animale optimale.

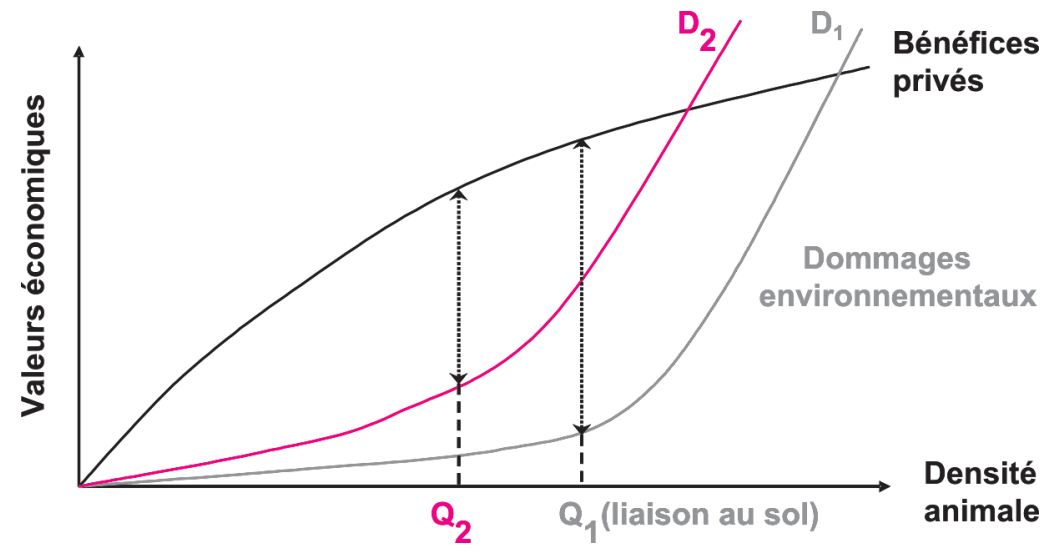

${ }^{2}$ Ce sont les masses d'eau qui feront l'objet d'un report de délai par rapport à l'objectif de bon état écologique en 2015, ou d'objectifs moins stricts. 
ble. Il y a bien ici l'idée de rechercher un bénéfice net et pas une amélioration à n'importe quel prix.

\section{2 / La récupération des coûts}

Le deuxième principe est le principe de récupération des coûts, dont la déclinaison environnementale est le principe pollueur-payeur. Le fait de donner un signal de prix aux ressources, services et pollutions a deux effets. D'une part, le signal de prix incite à ne pas gaspiller les ressources et les services, ainsi qu'à réduire les pollutions. D'autre part, il conduit à une utilisation efficace des ressources physiques et financières, en ce sens qu'il permet de maximiser le bénéfice global tiré d'un volume donné de ressource ou de service et de minimiser le coût global nécessaire pour réduire la pollution jusqu'à un niveau donné. Cette dernière propriété vient de ce que les coûts ou les bénéfices marginaux des différents agents sont égaux entre eux et au signal de prix.

Le principe pollueur-payeur est souvent confondu avec le principe de responsabilité environnementale, or il existe sur le plan économique des nuances importantes entre ces deux approches. Le principe pollueur-payeur prévoit que les frais résultant des mesures de prévention, de réduction et de lutte de la pollution doivent être pris en charge par le pollueur. Pour que ce principe fonctionne, il faut que le niveau de pollution acceptable soit fixé par le décideur public, par exemple à l'aide d'une norme ou d'un droit de propriété, assortis de pénalités dissuasives en cas de dépassement. Chaque pollueur recherche alors le moyen le moins coûteux d'atteindre la norme. Si les droits sont échangeables, l'ajustement des coûts individuels au prix de marché permet de minimiser le coût collectif pour respecter la norme ${ }^{3}$. Le principe pollueur-payeur est un principe de non subvention, bien que l'OCDE ait prévu des dérogations, notamment s'il s'agit de subventions non pérennes à des activités économiques en difficulté. La subvention à la dépollution a un effet incitatif pervers, en ce sens qu'elle fausse la perception des coûts par les agents et encourage les activités à fort contenu en pollution, voire la pollution elle-même (Baumol et Oates 1988). De plus, elle est un obstacle à la minimisation des coûts et permet de conserver des activités qui ne sont pas rentables quand on intègre l'environnement. A cet égard, elle est contraire au principe de durabilité.
Le principe de responsabilité environnementale introduit la notion de préjudice écologique, car au-delà de la prévention, il oblige également le responsable de dommages environnementaux à financer la réparation des dommages. Cette différence majeure avec le principe pollueur-payeur devrait en théorie inciter les agents à choisir d'eux-mêmes le niveau de pollution optimal, puisque le profit est désormais diminué du dommage environnemental, à condition que ce dernier soit correctement évalué. On parle alors de décentralisation de l'optimum, car le décideur public n'est plus obligé de fixer l'objectif environnemental, par exemple par la norme. A la figure 1, l'application d'un tel principe conduirait les producteurs à choisir spontanément les densités animales $\mathrm{Q}_{1}$ ou $\mathrm{Q}_{2}$ qui leur permettraient de maximiser leur profit, selon qu'ils seraient situés en zone banale ou sensible.

La DCE suggère d'utiliser des instruments économiques pour mettre en œuvre la récupération des coûts des services de l'eau, y compris les coûts pour l'environnement et les ressources. Elle précise que la politique de tarification de l'eau doit inciter les usagers à utiliser les ressources de façon efficace. Cela signifie que la tarification doit promouvoir une allocation optimale des ressources en eau entre les différents secteurs, de façon à maximiser la valeur collective. Conjointement, la tarification doit faire en sorte que la ressource attribuée à un secteur soit répartie entre les agents de façon à maximiser la valeur que le secteur en tire. Le raisonnement serait analogue pour la pollution : le signal de prix, taxe, permis ou autre, devrait maximiser la valeur collective nette et minimiser le coût de dépollution global. Cette utilisation efficace exige notamment que la récupération des coûts soit déclinée par secteur, en distinguant au moins l'industrie, les ménages et l'agriculture, comme le veut la DCE.

Malgré la réforme de la loi sur l'eau de 2006, ce principe n'est toujours pas appliqué en France. La redevance sur le prélèvement d'eau continue à être modulée selon les usages, domestiques ou économiques. Ceci explique que les prélèvements de l'agriculture, moins taxés que les autres usages, soient excessifs, malgré un système de quotas individuels, qui de surcroit ne répartit pas efficacement l'eau entre les agriculteurs. Plus généralement, ce sont les consommateurs d'eau qui paient pour les agriculteurs, pourtant responsables des prélèvements les plus importants et des pollutions les plus préoccupantes, ce qui freine l'utilisation efficace des ressources.

\section{3 / L’analyse économique de l'utilisation de l'eau}

Enfin, la DCE impose de procéder à une analyse économique de l'utilisation de l'eau dans chaque bassin hydrographique, dans le but de mettre en œuvre les principes d'optimalité et de récupération des coûts. Cette analyse porte sur la prévision à long terme de l'offre et la demande d'eau, tant en ce qui concerne les volumes, que les prix et les coûts des services liés à l'utilisation de l'eau, y compris les coûts pour l'environnement et les ressources. Cela signifie en particulier qu'il convient d'estimer les coûts de dépollution (offre) et la réduction de dommages (demande) impliqués par le bon état des eaux. Ces données sont nécessaires pour fonder les dérogations à l'objectif de bon état et la récupération des coûts. Il s'agit également d'élaborer des programmes combinant au moindre coût des mesures relatives aux utilisations de l'eau, dans l'esprit de l'analyse coût-efficacité.

\section{2 / Le dispositif français de maîtrise des pollutions des élevages}

\section{1 / Les mesures réglementaires}

Dans leur rapport sur la filière porcine française et le développement durable, commandé par les Ministères de l'agriculture et de l'écologie, Lessirard et Quevremont (2008) qualifient le dispositif français «d'édifice complexe relativement inefficace... aux résultats globaux décevants en matière de pollution diffuse... au regard des moyens engagés». Parmi les mesures qui se sont empilées successivement, on présente d'abord les mesures réglementaires, puis les mesures incitatives.

Le socle du dispositif est la réglementation sur les installations classées de 1976. Elle crée un régime de déclaration pour les petits élevages, alors que les élevages plus importants doivent obtenir une autorisation sur la base d'une étude d'impact sur l'environnement. Le corps du dispositif se focalise sur les pollutions azotées, avec la direc-

\footnotetext{
${ }^{3}$ La taxe est une autre façon d'appliquer le principe pollueur-payeur, qui conduit aussi à un coût collectif minimisé, car elle joue le rôle d'un signal de prix.
} 
tive nitrate de 1991, qui vise à l'équilibre de la fertilisation, quelle que soit l'origine de l'azote. Elle est appliquée sous forme de programme d'action quadriennaux, qui obligent les agriculteurs à adopter des bonnes pratiques agricoles et un calendrier d'épandage, à élaborer un plan de fumure prévisionnel et tenir un cahier de fertilisation, à limiter les apports d'azote organique des animaux à $170 \mathrm{~kg}$ par hectare épandable, etc. Dans les Zones à Actions Complémentaires (ZAC), qui correspondent aux têtes de bassin versant en amont des prises d'eau potable, c'est la fertilisation azotée totale qui est plafonnée (210 kg par ha). Annexés aux programmes d'action, les programmes de résorption concernent les Zones d'Excédents Structurels ${ }^{4}$ (ZES). Les grandes exploitations ont l'obligation de traiter leurs excédents. Les autres exploitations ont accès aux terres d'épandage disponibles dans la limite de plafonds, au dessus desquels elles doivent également traiter ou transférer leurs excédents. Sont également prévues l'interdiction de transfert vers les cantons à plus de $140 \mathrm{~kg}$ d'azote par hectare et l'interdiction de création ou d'extension d'élevages en ZES comme en $\mathrm{ZAC}^{5}$.

Néanmoins, pour que les élevages situés en ZES puissent évoluer, un dispositif autorisant les restructurations internes (entre ateliers d'espèces différentes sur un même site) ou externes (entre sites situés en ZES) est créé en 2003. Les regroupements se font sur la base des émissions azotées, à azote constant (en interne) ou avec prélèvement d'azote par l'Etat pouvant aller jusqu'à $40 \%$ (en externe). Ce dispositif est assez critiquable dans la mesure où il crée des quotas d'azote échangeables entre exploitations, qui présentent le double inconvénient de ne pas être liés à la terre et de créer des rentes inéquitables en faveur des producteurs en place. Il s'agit en fait de droits à produire, inefficaces sur le plan environnemental puisqu'ils accentuent la concentration locale des animaux (voir Mahé et Ortalo-Magné 2001). Ce système très lourd au plan administratif permet cependant à l'Etat de contenir, voire de réduire mécaniquement la pression azotée globale en ZES, en faisant financer le dispositif par les producteurs euxmêmes.

Aux zonages existants est venu s'ajouter en 2007 celui des bassins versants en contentieux avec l'Europe, car non conformes à la directive de 1975 sur les eaux superficielles destinées à la production d'eau potable 6 . Dans ces bassins versants, la fertilisation azotée totale par hectare de SAU est plafonnée à $140 \mathrm{~kg}$ pour les exploitations porc/ céréales, $160 \mathrm{~kg}$ pour les exploitations de polyculture élevage bovin et $170 \mathrm{~kg}$ pour les exploitations légumières.

Lessirard et Quevremont (2008) montrent bien comment cet arsenal réglementaire constitue une obligation de moyens, mais pas une obligation de résultats. Leur rapport pose notamment la question majeure du contrôle du respect des plafonds de fertilisation azotée. Baron et al (2001) avaient déjà montré que la législation sur les épandages et le prêt de terres ne s'appliquait pas avec la rigueur nécessaire. En raison de l'instabilité des contrats d'épandage dans le temps, Lessirard et Quevremont (2008) proposent de demander une déclaration annuelle aux parties. Par ailleurs, bien que les plafonds puissent porter également sur l'azote minéral en ZAC et dans les bassins en contentieux, il n'existe pas d'outil opérationnel pour contrôler ou réduire le minéral. Le contrôle de la comptabilité permettrait d'y pallier.

\section{2 / Les mesures incitatives}

De nombreux programmes incitatifs se sont déroulés depuis 1990. Créé en 1993, le PMPOA a financé les deux tiers du coût de la mise aux normes des bâtiments d'élevage (récupération et stockage des déjections animales). Les systèmes d'alimentation biphase et multiphase, qui permettent de réduire les rejets azotés, ont également été encouragés, comme l'avaient suggéré Dourmad et al (1995). Les contrats territoriaux d'exploitation, puis les contrats d'agriculture durable ont été mobilisés pour infléchir les pratiques agricoles, par exemple en encourageant financièrement la substitution des engrais organiques aux engrais minéraux. Le plan d'action pour le développement pérenne de l'agriculture bretonne de 2002 prévoyait de financer en partie la résorption de 80000 tonnes d'azote, dont 60000 tonnes par traitement du lisier (30 à 60\% des investissements couverts par l'Etat, les collectivités et l'agence de l'eau). Les programmes d'action relatifs aux bassins versants en contentieux prévoient une aide au traitement du lisier, au transfert des effluents et à la réduction des chep- tels. Des mesures agri-environnementales, puis des indemnités compensatrices de contrainte environnementale dégressives sont prévues pour compenser les baisses de rendement et les surcoûts de gestion des effluents, dans ces bassins versants où les plafonds de fertilisation imposés sont bas.

En appui de ces programmes incitatifs, trois programmes Bretagne Eau Pure (BEP) se sont succédés entre 1990 et 2006. Organisé selon la logique des bassins versants, le programme BEP vise notamment à accélérer l'application de la réglementation dans le secteur agricole. Les instruments de BEP sont l'action collective, la sensibilisation, la formation, la démonstration, les diagnostics de pratiques agricoles et les engagements contractuels pour les agriculteurs les plus motivés. Il est attendu un effet d'amplification des modifications de pratiques, dû à l'essaimage des comportements vertueux à partir des bassins versants BEP.

BEP repose fondamentalement sur le volontariat des agriculteurs, ce qui l'apparente à ce que l'OCDE appelle les «programmes volontaires publics». Or la littérature internationale sur les approches volontaires indique que leur efficacité environnementale est faible, en raison du rôle central de l'industrie, des engagements non exécutoires et de l'absence de contrôle (OCDE 1999 et 2003). Elles peuvent même être utilisées pour ne pas respecter la réglementation ou bloquer son durcissement (détournement de la réglementation). Aux Etats-Unis, une analyse du secteur agricole menée par l'US Department of Agriculture (USDA) conclut que l'éducation n'est probablement pas efficace, quand la recherche de l'optimum environnemental implique la réduction du profit des producteurs, même quand ceux-ci comprennent la relation entre leurs pratiques et la qualité de l'eau (Ribaudo et al 1999).

Une étude du Service Régional de Statistique Agricole de Bretagne de 2005 montre que BEP améliore les pratiques quand il existe une menace réglementaire crédible et/ou un programme d'aides publiques, ou quand les actions ne sont pas douloureuses financièrement (Cébron et al 2008). En revanche, la couverture des sols en hiver ne dépend pas de BEP, mais est plus importante en ZAC où il existe une

\footnotetext{
4 Cantons où les apports d'azote organique dépassent $170 \mathrm{~kg}$ par hectare épandable.

5 Sauf jeunes agriculteurs et élevages de dimension économique insuffisante.

6 Concentration en nitrate supérieure à $50 \mathrm{mg}$ par litre.
} 
obligation réglementaire et une indemnité compensatrice. Enfin, BEP n'a pas d'effet sur le niveau d'azote minéral, probablement parce que ce dernier n'est pas contrôlé, comme on l'a vu, et que son remplacement par des engrais organique implique des coûts.

Parmi les mesures incitatives, on trouve également la redevance pollution des élevages, volet répressif du PMPOA, créée en 1993 dans le but d'accélérer la mise aux normes des élevages et effectivement appliquée à partir de 1999. Elle était assise initialement sur la qualité de récupération des déjections, améliorable grâce aux aides du PMPOA, et sur le chargement animal. Ce dernier critère permettait, sans le dire ni le faire vraiment rigoureusement, de pénaliser le dépassement de la norme des $170 \mathrm{~kg}$ d'azote organique par hectare, c'est-à-dire les exploitations en excédent. La loi sur l'eau de 2006 a modifié l'assiette de cette redevance. Elle est désormais assise sur tous les UGB de l'élevage et plus seulement sur les Unités Gros Bovins (UGB) excédentaires, même si les élevages dont le chargement est inférieur à 1,4 UGB par hectare en sont exonérés ${ }^{7}$ (élevages extensifs, notamment de montagne). Bien que le taux de taxe par unité d'azote ait été divisé par un facteur proche de 8 , la recette globale n'est pas modifiée car le paiement de la redevance est mutualisé sur un plus grand nombre d'élevages, qu'ils soient excédentaires ou pas. En Bretagne, cela signifie que les élevages liés au sol et les élevages hors-sol ayant résorbé leurs excédents paieront pour les élevages excédentaires ${ }^{8}$. Il s'agit d'un retour en arrière en matière d'application du principe pollueur-payeur, qui aura des conséquences négatives sur l'équité entre les producteurs et la pédagogie de l'environnement, ainsi que sur l'incitation économique à ne pas polluer, alors qu'elle était déjà faible. D'une manière générale, la nouvelle loi sur l'eau se limite à compter sur le volontariat des acteurs et les aides publiques pour faire reculer les pollutions diffuses d'origine agricole.

\section{3 / Analyse économique du dispositif français}

Cette recension montre que la réglementation est l'instrument privilégié de la politique française de maîtrise des pollutions des élevages. Cependant, il reste des problèmes importants quant à la définition des normes censées limiter les pollutions diffuses et quant à la vérification de leur respect par les agriculteurs. Les plafonds de fertilisation totale ne sont pas généralisés et le contrôle des épandages et de la consommation de minéral est inefficace ou impossible. Par ailleurs, on observe que les prescriptions réglementaires vont bien audelà de la poursuite d'objectifs environnementaux, puisqu'elles portent aussi sur le choix des technologies de dépollution, sur la répartition des surfaces d'épandage, sur la limitation et la restructuration de la production, etc. En imposant des contraintes non justifiées sur le plan environnemental, on risque de créer des inefficacités économiques et de décourager inutilement les entrepreneurs, sans garantie d'efficacité environnementale. En outre, la multiplication des réglementations alourdit considérablement les missions de contrôle de l'administration et l'empêche de se concentrer sur la question essentielle de la vérification des normes environnementales.

Le dispositif a eu massivement recours aux subventions, sans lesquelles on n'observe pas de modification des pratiques agricoles. Il enfreint donc le principe pollueur-payeur et se trouve en contradiction avec la DCE. Lessirard et Quevremont (2008) démontrent d'ailleurs que l'administration française reste attachée à cette stratégie, puisqu'ils proposent de mutualiser une cotisation perçue à l'abattage pour pérenniser les aides aux investissements environnementaux, malgré l'encadrement communautaire des aides. Dans le même esprit, on constate que la redevance pollution des élevages, qui constituait un timide début d'application du principe pollueur-payeur, a fini par être démantelée et mutualisée elle aussi. Cette approche mutualiste de la politique de l'eau en France avait déjà été dénoncée dans les rapports sur le fonctionnement des agences de l'eau (cf. infra). Ce refus persistant d'appliquer le principe pollueur-payeur s'explique par la volonté de maintenir la production, même si elle n'est pas durable, afin d'éviter les ajustements structurels dans la filière. Il a pour contreparties l'inefficacité économique et l'inflation réglementaire dans les ZES, afin de tenter d'y endiguer la croissance des effectifs.
La conséquence est que les éleveurs ne sont pas incités à rechercher les méthodes de résorption les moins coûteuses, au premier rang desquelles on trouve l'épandage et la réduction du minéral, puisqu'ils ne sont pas confrontés aux coûts réels (voir Mahé et Le Goffe 2002). Le Goffe et Salanié (2005) ont notamment montré comment la subvention au traitement du lisier incite les agriculteurs à utiliser davantage d'azote minéral, ce qui explique en partie pourquoi sa consommation ne baisse plus en Bretagne depuis 2002, malgré les actions de BEP. Cette sous-utilisation de l'épandage s'explique également par les contraintes réglementaires qui obligent certains élevages à traiter, plafonnent les surfaces d'épandage ou limitent les transferts. Par ailleurs, les éleveurs n'ont pas le signal d'arrêter de produire, quand le coût de résorption supplémentaire devient supérieur à la valeur créée en plus. Cela conduit à pérenniser des exploitations qui ne sont pas durables et incite à la croissance des excédents.

A l'échelle sectorielle, le coût global n'est pas minimisé, comme l'ont montré Djaout et al (2008), à l'aide d'un modèle linéaire de gestion des effluents d'élevage sous contrainte de la directive nitrate. La simulation effectuée pour le département des Côtes d'Armor montre que le coût global de résorption serait divisé par deux, si on appliquait le principe pollueur-payeur plutôt que le Plan d'action pour le développement pérenne de l'agriculture bretonne. L'application du principe pollueurpayeur et de la norme stimulerait le marché de l'épandage, qui ne fonctionne pas bien aujourd'hui en Bretagne, où une proportion importante de terres ne reçoit pas d'engrais organique. Le signal de prix du service d'épandage inciterait les élevages les plus efficaces à traiter leurs effluents, alors que les élevages les moins efficaces privilégieraient l'épandage ou la réduction du cheptel et que les agriculteurs auraient intérêt à utiliser les engrais organiques à la place des engrais minéraux.

\section{3 / Les expériences étran- gères}

Peut-on étendre ce constat aux autres pays européens ? Fermin (2007) a comparé l'application de la directive

\footnotetext{
${ }^{7}$ L'argument de la simplification administrative, avancé pour la modification de l'assiette, n'est pas recevable dans la mesure où, pour l'exonération, il faut continuer à déterminer le chargement, base de l'assiette antérieure.

8 En Bretagne, la majeure partie de l'excédent d'azote régional provient des exploitations hors-sol de porcs et de volailles (Cébron et al 2008).
} 
nitrate en Bretagne et dans trois pays $\mathrm{du}$ nord de l'Europe (Pays-Bas, Danemark, Flandres belges), confrontés aux mêmes enjeux de la concentration des élevages. Par rapport à la Bretagne, la densité animale y est supérieure aux Pays-Bas, comparable en Flandres, inférieure au Danemark. Dans ces trois pays ou région, ce qu'on appelle «la politique du lisier» a commencé à la fin des années 80 , c'est-àdire plus tôt qu'en France, et a connu des évolutions importantes suite aux échecs des programmes successifs, pour aboutir à la forme actuelle. L'évolution a été particulièrement laborieuse aux Pays-Bas, où le système de comptabilité minérale MINAS adopté en 1998, qui permettait de taxer les pertes du bilan minéral entrées-sorties, a finalement été abandonné en 2006 en raison des coûts administratifs et judiciaires et des contestations européennes. A l'opposé, le Danemark est considéré comme le bon élève de l'Europe, notamment en raison de sa règle d'harmonie qui organise la liaison de l'élevage au sol depuis 2002, ce qui lui a valu d'obtenir une dérogation à la directive nitrate pour les élevages bovins à l'herbe (230 kg d'azote organique/ha). Dans ce pays, la production porcine augmente mais la pression azotée reste constante, car les volailles et surtout les bovins sont en diminution.

Les politiques des trois pays ont progressivement convergé vers ce qu'on pourrait appeler le «modèle danois», qui est conforme à la DCE. Des plafonds de fertilisation totale en azote et phosphore sont fixés par culture et par type de sol. Les transports et les échanges de lisier font l'objet de déclarations systématiques à l'administration. Le rapprochement de ces déclarations permet de cibler les contrôles sur les exploitations à risque. Des amendes dissuasives et/ou des sanctions pénales sont décidées en cas de dépassement ou de fraude. Le taux des amendes par $\mathrm{kg}$ d'azote au dessus des plafonds est environ 10 fois supérieur à celui de l'ancienne redevance pollution des élevages en France, ce qui permet une véritable application du principe pollueur-payeur. A la différence du système MINAS, ou de la redevance pollution des élevages en France, il n'y a pas de taxation systématique des élevages, ce qui permet d'économiser des coûts administratifs. Le traitement du lisier n'a pas été subventionné et est donc peu utilisé ${ }^{9}$. En conséquence, le marché de l'épandage est très actif, d'autant plus que des intermédiaires publics ou privés organisent la rencontre des vendeurs et des acheteurs, ce qui permet de diminuer les coûts de transaction.

Les Pays-Bas et les Flandres ont néanmoins conservé des quotas animaux exprimés en équivalent phosphore et échangeables entre exploitations. Ces mesures, qui ont inspiré celle sur la restructuration des élevages bretons en ZES, permettent le prélèvement à l'occasion des échanges ou le rachat des quotas par l'Etat. On a vu cependant qu'elles constituaient un pis-aller, que ce soit sur le plan environnemental ou économique. En maîtrisant précocement sa densité animale et en évitant les dérapages ultérieurs, le Danemark a su se passer de cette approche pour se concentrer sur le respect des plafonds de fertilisation.

\section{Conclusion}

Dans sa tentative de faire appliquer la directive nitrate, la France ne s'inspire pas des principes économiques inscrits dans la DCE. Le dispositif privilégie la réglementation ainsi que l'éducation et le volontariat des producteurs, au détriment des instruments économiques incitatifs. On constate le refus persistant d'appliquer le principe pollueurpayeur : les investissements de stockage et de traitement des effluents ont bénéficié de subventions importantes et la redevance pollution des élevages, seul instrument qui frappait faiblement les excédents d'azote, a été démantelée. La récupération des coûts par secteur est mal réalisée, dans la mesure où ce sont surtout les consommateurs d'eau et peu les éleveurs qui financent la mise aux normes des élevages. Ceci explique que les producteurs ne soient pas incités à utiliser les méthodes de résorption des excédents les moins coûteuses et que le coût global, non minimisé, soit trop élevé au regard des résultats environnementaux obtenus.

Le dispositif breton des bassins versants en contentieux est celui qui se rapproche le plus du modèle danois, par la fixation de plafonds de fertilisation azotée totale différenciés par systèmes de production, qui font l'objet de contrôles renforcés. Certes, il a encore recours aux aides publiques, mais certaines sont dégressives sur cinq ans, laissant présager le proche avènement $\mathrm{du}$ principe pollueur-payeur et ses conséquences en terme de valorisation de l'épandage et de minimisation des coûts. Dans une étude réalisée pour le Bureau agriculture-environnement de la DGTPE du Ministère de l'économie, Fernandes (2008) fait des propositions qui permettraient de lever les freins au développement des marchés de l'épandage en France. La mission de l'Etat devrait se concentrer sur la définition, le contrôle et l'application rigoureuse de plafonds de fertilisation totale (azote et phosphore) généralisés et du principe pollueur-payeur. La réglementation ZES serait considérablement allégée, en supprimant l'obligation de traitement, les plafonds d'épandage, l'interdiction de création ou d'extension d'élevage, l'interdiction d'exportation d'effluents dans les cantons à plus de $140 \mathrm{~kg}$ d'azote/ha. La législation sur les installations classées serait modifiée pour permettre l'évolution temporelle des plans d'épandage. Enfin, le recours à des gestionnaires publics ou privés du marché de l'épandage serait encouragé.

Il resterait encore à traiter le problème des pollutions accidentelles dues au stockage du lisier, récurrentes en Bretagne, qui ne relèvent pas de la directive nitrate. Comme il s'agit de gestion des risques, cela pourrait passer par l'assurance obligatoire de la responsabilité environnementale des élevages, à la manière du traitement préventif des marées noires aux EtatsUnis. On peut penser que l'application progressive du principe de responsabilité environnementale à l'agriculture conduira à une estimation à la hausse du préjudice écologique, ce qui incitera les assurances à réduire les risques de pollution accidentelle par la sécurisation des installations et à augmenter les primes pour les systèmes d'élevage à risques pour l'eau ${ }^{10}$. A terme, cette internalisation des risques environnementaux par l'assurance pourrait compléter les mesures visant à appliquer la directive nitrate et contribuer à rendre l'élevage plus durable.

Enfin, précisons que seules les questions de pollution des eaux et la dimension économique ont été traitées dans cet article. Dans une acception complète du concept de durabilité, il conviendrait de considérer les interactions avec les autres compartiments écologiques et les questions sociales liées aux nécessaires ajustements structurels induits par les politiques environnementales.

\footnotetext{
9 Cependant des aides à la valorisation énergétique de la biomasse ont été accordées récemment, au titre des énergies renouvelables et de la lutte contre l'effet de serre.

${ }^{10}$ Les systèmes à base de lisier comportent plus de risques de pollutions accidentelles pour l'eau que les systèmes à base de fumier.
} 


\section{Références}

Anonyme, 2000. Directive $n^{\circ}$ 2000/60/CE du Parlement européen et du Conseil du 23 octobre 2000 établissant un cadre pour une politique communautaire dans le domaine de l'eau. Journal officiel des communautés européennes $n^{\circ}$ L 327 du 22 décembre 2000.

Anonyme, 2002. Plan d'action pour un développement pérenne de l'agriculture et de l'agroalimentaire en bretagne et pour la reconquête de la qualité de l'eau : le financement de l'Etat. DRAF Bretagne, 28 janvier, 3 p.

Aurousseau P., 2008. Diagnostic de l'évolution de la qualité des eaux dans les bassins versants de Bretagne. Colloque «L'eau et le droit», AFDR Bretagne, 10 octobre, Rennes, France.

Baron P., Barthélémy F., Bouvier M., Martin X., Vogler J.P., 2001. Elevages et fonctionnement du Conseil Départemental d'Hygiène en Ille et Vilaine. Ministère de l'aménagement du territoire et de l'environnement, Paris,France, Baumol W.J., Oates W.E., 1988. The theory of environmental policy. Cambridge University Press, Cambridge, UK

Cébron D., Michel P., Hurvois Y., Leroy S., 2008. Gestion globale de la fertilisation en 2004 : Les excédents azotés ont diminué depuis 2000. Agreste Bretagne, mars, 1-8.

Commissariat général au Plan, 1997. Evaluation du dispositif des agences de l'eau : rapport au Gouvernement. La Documentation française, Paris, France.

Cour des comptes, 1997. La Gestion des services publics locaux d'eau et d'assainissement : rapport public particulier. Direction des journaux officiels, Paris, France.
Cour des comptes, 2002. La préservation de la ressource en eau face aux pollutions d'origine agricole : le cas de la Bretagne : rapport au Président de la République suivi des réponses des administrations et des organismes intéressés. Direction des journaux officiels, Paris, France.

Courtecuisse A., Davy T., Laurans Y., Rideau J.P., Rinaudo J.D., Strosser P., 2002. Quel rôle pour l'économie dans la Directive Cadre sur l'Eau ? Un processus, une approche, des outils, un guide. Colloque SHF «Eau et économie», septembre, Paris, France.

Djaout F., Le Goffe P., Tauber M., 2008. Comment appliquer la directive nitrates et à quel coût ? Une modélisation spatiale du marché de l'épandage. A paraître dans Economie et Prévision, 39p.

Dourmad J.Y., Le Mouël C., Rainelli P., 1995. réduction des rejets azotés des porcs par la voie alimentaire : évaluation économique et influence des changements de la Politique Agricole Commune. INRA Prod. Anim., 8, 135-144.

Fermin N., 2007. Sous la direction de P Le Goffe. Application de la directive «nitrates» dans trois pays européens : Pays-Bas, Danemark, Flandres. Rapport pour la Préfecture de Région Bretagne, $85 \mathrm{p}$.

Fernandes L., 2008. Comment réduire la pollution azotée en France ? Propositions de développement des marchés de droits d'épandage des effluents d'élevage. Mémoire de fin d'études d'ingénieur agronome de l'ENSAR

IFEN, 2006. L'environnement en France. Institut Français de l'Environnement, Paris, France.
Lessirard J., Quevremont P., 2008. La filière porcine française et le développement durable. Rapport de mission pour les Ministères de l'écologie et de l'agriculture, Paris, France, $74 \mathrm{p}$. + annexes.

Le Goffe, P., J. Salanié., 2005. Le droit d'épandage a-t-il un prix ? Mesure sur le marché foncier. Cahiers d'Economie et Sociologie Rurales $77: 36-63$

Mahé L. P., Le Goffe P., 2002. La résorption des excédents d'épandage : principes économiques d'un plan d'action pour la Bretagne, Rapport à la Préfecture de la Région Bretagne, $63 \mathrm{p}$.

Mahé L.P., Ortalo-Magné F., 2001. Politique agricole : un modèle européen. Presses de Sciences Po, Paris, France, 235p.

OCDE, 1999. Les approches volontaires dans les politiques de l'environnement. OCDE, 163p.

OCDE, 2003. Voluntary approaches for environmental policy. Effectiveness, efficiency and usage in policy mixes. OCDE, 143p.

Ribaudo M.O., Horan R.D., Smith M.E. 1999. Economics of water quality protection from nonpoint sources : theory and practice. Resource Economics Division, Economic Research Service, US Department of Agriculture. Agricultural Economic Report, 782.

Villey-Desmeserets F.., Ballay D., Tricard D., Henry de Villeneuve C., 2001. La Politique de préservation de la ressource en eau destinée à la consommation humaine : rapport d'évaluation du Commissariat général du Plan. La Documentation française, Paris, France.

\section{Résumé}

En Bretagne, les résultats de la politique de l'eau ne sont pas à la hauteur des sommes engagées par les éleveurs et les pouvoirs publics. L'explication que propose l'article est que les principes économiques inscrits dans la Directive Cadre sur l'Eau (DCE) ne sont pas appliqués dans notre pays, contrairement à ce qu'on observe dans les pays du nord de l'Europe. Dans la DCE, l'objectif environnemental est défini comme un optimum économique, qui doit maximiser la valeur collective ou minimiser les coûts sociaux. En outre, la DCE donne les moyens d'atteindre cet optimum, en imposant la "récupération des coûts ", dont la version environnementale est le principe pollueurpayeur (PPP). Ces concepts sont confrontés au dispositif français de maîtrise des pollutions des élevages, qui se caractérise par l'hypertrophie de la réglementation et le refus d'appliquer le PPP. C'est ce qui explique que les producteurs ne soient pas incités à réduire la pollution en employant les solutions les moins coûteuses, au premier rang desquelles se trouve l'épandage. En conclusion, on suggère de recentrer les missions de l'Etat sur la définition des normes et l'application du PPP, et d'alléger la réglementation de manière à lever les freins à l'épandage.

\section{Abstract}

\section{Water Policy : An economic approach and application to farm pollution}

In Brittany, water policy gives little results compared to the money involved. Compared to northern European countries the French government does not enforce the economic principles of the water framework directive (WFD). The WFD defines the environmental target as an economic optimum that maximises the collective value or minimises social costs. Further, the WFD states that the water policy has to enforce cost recovery and the polluter-pay principle (PPP). The WFD economic principles are compared to the French policy to reduce animal pollution which is based on a hypertrophic set of regulation and rejection of the PPP. This explains why producers do not have incentives to reduce pollution by using least cost solutions like manure spreading. We suggest limiting the state's missions missions to defining and enforcing standards via the PPP and to reducing regulations therefore lifting the obstacles to manure spreading.

Le GOFFE P., 2008. La politique de l'eau : approche économique et application à la pollution des élevages. INRA Prod. Anim., 21, 419-426. 
